\title{
Climate change consensus messages may cause reactance in conservatives, but there is no meta-analytic evidence that they backfire
}

\author{
Jacob B. Rode ${ }^{1}$, Amy L. Dent ${ }^{2}$, and Peter H. Ditto ${ }^{2}$ \\ ${ }^{1}$ Department of Psychology, Harvard University \\ ${ }^{2}$ Department of Psychological Science, University of California, Irvine
}

\begin{abstract}
Author Note
Jacob B. Rode (iD https://orcid.org/0000-0001-8289-7760
\end{abstract}

All data and study materials are publicly available at: https://osf.io/a39hv/. We have no known conflict of interest to disclose.

Correspondence concerning this article should be addressed to Jacob Rode, Department of Psychology, Harvard University, William James Hall, 33 Kirkland Street, Cambridge, MA 02138. Email: jbenjaminrode@gmail.com

This is an original manuscript of an article published by Taylor \& Francis in Environmental Communication on August 26, 2022, available online:

http://www.tandfonline.com/10.1080/17524032.2022.2101501 


\begin{abstract}
Recent studies suggest climate change consensus messages may cause psychological reactance for conservatives. However, it remains unknown how much this reactance impacts the effectiveness of consensus messaging. Using data from a recent meta-analysis on climate change interventions, the current paper seeks to add context to the debate over reactance. We integrated data from 20 experiments ( $N=19,200$ participants) that test how consensus messages (compared to a control condition) impact climate change attitudes. The effect of consensus messages on attitudes was small yet statistically significant and positive $(g=0.09)$, and not significantly moderated by political affiliation. Moreover, the moderating role of political affiliation was similar for consensus messages compared to other interventions for climate change attitudes. While conservatives may experience resistance to consensus messages, there is little metaanalytic evidence that consensus messages backfire.

Keywords: consensus messaging, psychological reactance, climate change, meta-analysis
\end{abstract}


Recent polling data suggests that $58 \%$ of Americans agree "most scientists" think that global warming is happening, and about $20 \%$ of Americans say that over $90 \%$ of climate scientists think that human-caused global warming is happening (Leiserowitz et al., 2020). Although these percentages have been increasing over the past decade, public opinion still falls short of the actual percentage of climate scientists who believe that human-caused global warming is happening, popularized as the $97 \%$ consensus (Cook et al., 2013). Because of this discrepancy, scholars have used scientific consensus as a tool to influence climate change attitudes, with evidence that consensus messages have a substantial effect on perceived scientific consensus and smaller impacts on downstream climate change attitudes (i.e., a cascading effect; van der Linden, 2021). However, there are still theoretical and methodological questions about the effectiveness of consensus messaging (Landrum \& Slater, 2020).

Recent research has found that consensus messages may spark psychological reactance, which is a psychological state that typically includes anger and counterarguing in response to attempted persuasion (Rains, 2013). Specifically, Ma et al. (2019) found that climate skeptics, and particularly those who identify as Republican or Independent, report increased feelings of psychological reactance in response to a climate change consensus message. Moreover, Ma et al. (2019) reported a significant indirect effect of consensus message on support for mitigation policy among Republican and Independent climate skeptics, where the consensus message (compared to no message) led to less support for policy via increased reactance. In response, van der Linden et al. (2019) argued that consensus messages do not cause psychological reactance, though they found that conservatives saw consensus messages as more manipulative than did liberals (for more context and a counter response, see Dixon et al., 2019). Recently, Chinn and Hart (2021) sought to adjudicate the debate on reactance by testing different designs (used by the 
two previous studies). They found that participants who saw consensus messages reported more psychological reactance than those who did not see any message, and importantly, that the effect was driven by Republican participants. A response to this most recent paper argues that their results may not be strong evidence of reactance, pointing out the limited effect sizes of the political interaction (van der Linden et al., 2021). Taken together, these recent findings provide some evidence that seeing a consensus message leads conservatives to feel psychological reactance, but the strength and even presence of this reactance is still debated (for a longer discussion about how to reconcile the discrepant findings, see Bayes et al., 2020).

While compelling, the reactance debate leads to a few questions. What are the implications of increased psychological reactance? If consensus messages spark reactance, what effect does this reactance have on climate change attitudes? Though Ma et al. (2019) report a significant negative indirect effect of consensus message on policy support, the total effect of consensus message on policy support is unknown. Additionally, none of the other studies on reactance report impacts of the consensus message on climate change attitudes, like support for policy. As van der Linden et al. (2021) note in their response to Chinn and Hart (2021), the importance of reactance is dependent upon how consensus messages ultimately impact climate change attitudes.

To help answer the question about the implications of psychological reactance, we take advantage of our recent meta-analysis that investigates the effectiveness of interventions for climate change attitudes (Rode, Dent, et al., 2021). Though we included a variety of intervention types in the meta-analysis (e.g., emotion, morality), consensus messaging had the most independent samples (20). Therefore, we use these data to investigate: 1 ) the overall effect of consensus messages on climate change attitudes, 2) if consensus messages are less effective for 
conservatives than for liberals, and 3) if consensus messages are particularly effective or ineffective compared to other types of interventions. The answers to the second and third questions allow us to contextualize the extent of the problem of psychological reactance: How much harm is being caused by consensus messages? If the psychological reactance caused by these messages is problematic, then we would expect consensus messages to be less effective for conservatives than for liberals and potentially even backfire (i.e., lead to worse outcomes for conservatives than no message). Additionally, we might expect consensus messages to be particularly ineffective for conservatives compared to other types of interventions. We analyze and interpret these data to help contextualize the implications of psychological reactance from consensus messages.

\section{Method}

The full details of the meta-analysis can be found in the original paper (Rode, Dent, et al., 2021). Here, we report on the details related to consensus messaging specifically. There were three inclusion criteria for the meta-analysis: 1) random assignment of participants into study conditions, 2) a control condition where participants did not receive an intervention related to climate change, and 3) a measure of climate change attitudes after the experimental manipulation. Because the focus of the meta-analysis was on climate change attitudes in the U.S., studies were excluded if they did not include U.S. participants exclusively. The search was conducted in 2019 and early 2020, with the population of studies limited to those available in 2019 or earlier.

Included studies were coded for a variety of characteristics, including intervention type. Two trained coders described each intervention and placed it into one of several pre-specified categories. Relevant for the current analysis, many interventions $(21 \%)$ were classified as 
consensus-based messages. For analyses, we excluded interventions that provided misinformation, or messages designed to lead to climate change skepticism (labeled as negative interventions in the original paper). Additionally, we excluded interventions that included both misinformation and consensus messages (labeled as mixed interventions in the original paper). Therefore, all interventions in the current analysis included a consensus message, and some of the interventions included additional information (full dataset: https://osf.io/a39hv). ${ }^{1}$

To test the effect of political affiliation, effect sizes were calculated separately for the full sample, liberals/Democrats, conservatives/Republicans, and moderates/Independents. When studies reported both political party and ideology, we used political party in the effect size calculation. Though the measure of political affiliation includes effect sizes calculated based on either political party or ideology, we will refer to the three groups as liberals, conservatives, and moderates.

In the full meta-analysis, studies included a range of types of climate change attitudes. In the analysis of consensus studies, most effect sizes consisted of a measure of belief that climate change is happening (18 effect sizes, 19\%), belief that climate change is human-caused (28 effect sizes, 30\%), risk (18 effect sizes, 19\%), or support for policy (18 effect sizes, 19\%). Fewer studies included measures of concern or worry (9 effect sizes, 10\%) and other attitudes (2 effect sizes, $2 \%$ ).

\section{Analyses}

Effect sizes were calculated as the average climate change attitude score for the intervention group (consensus message) minus the control group (no consensus message), calculated with Hedges' $g$ to adjust for small samples (Lakens, 2013). Effect sizes were calculated such that stronger positive effect sizes corresponded to stronger climate change 
attitudes in the consensus message group compared to control (e.g., more belief). Because studies could include multiple treatments (sharing the same control condition) and multiple dependent variables (same people responding to multiple dependent variables), the calculated effect sizes were not independent. Therefore, we used robust variance estimation (RVE) to account for these dependent effect sizes, using a correlated effects model and the corresponding weighting scheme (Hedges et al., 2010). For moderation by political affiliation, we used the Approximate Hotelling-Zhang (AHZ) test (Tipton \& Pustejovsky, 2015). In RVE, the estimate of between-study variance $\left(T^{2}\right)$ assumes a common correlation; we used the default correlation of .80 but report sensitivity tests (i.e., using correlations of 0 and 1) in the Supplemental Materials (the pattern of results is the same). We analyzed the data using the robumeta package in $\mathrm{R}$ (Fisher \& Tipton, 2015).

\section{Results}

A total of 16 reports included consensus message interventions, consisting of 20 independent samples, 93 effect sizes (before separating by political affiliation), and 19,200 participants. Overall, consensus messages had a small but statistically significant positive effect on attitudes, $g=0.09,95 \% \mathrm{CI}[0.05,0.13], t(4.40)=5.58, p=.004$. This effect size is similar to that of other interventions aimed at climate change attitudes (Rode, Dent, et al., 2021). Importantly, political affiliation was not a significant moderator of effect size for consensus message interventions, $F(6.65)=0.89, p=.45$ (Figure 1$).^{2}$ There was not a statistically significant difference between the average effect sizes for liberals and conservatives, $b=0.05$, $95 \%$ CI $[-0.03,0.12], t(7.65)=1.41, p=.20$.

To investigate how consensus messages compare to other interventions, we calculated the estimated effect sizes separated by political affiliation for consensus studies as well as for all 
interventions in the original meta-analysis (excluding consensus studies). If consensus messages uniquely spark reactance in conservatives, we might expect that the effect of political affiliation differs between consensus interventions and other interventions. However, the pattern of effect sizes for non-consensus interventions is the same: effect sizes were slightly larger for liberals ( $g$ $=0.082)$ than for conservatives $(g=0.076)$, but this political difference was not statistically significant, $b=0.01,95 \%$ CI $[-0.07,0.08], t(26.52)=0.16, p=.88$ (Table 1$)$. To formally test how consensus messages compare to other interventions, we used the full dataset (including all interventions) and conducted a meta-regression to test the interaction of political affiliation and intervention type (dummy coded with consensus messages as 1 and all other interventions as 0 ). There was not a significant interaction effect, $F(14.00)=0.75, p=.49$. 


\section{Figure 1}

Effect Sizes Separated by Political Affiliation

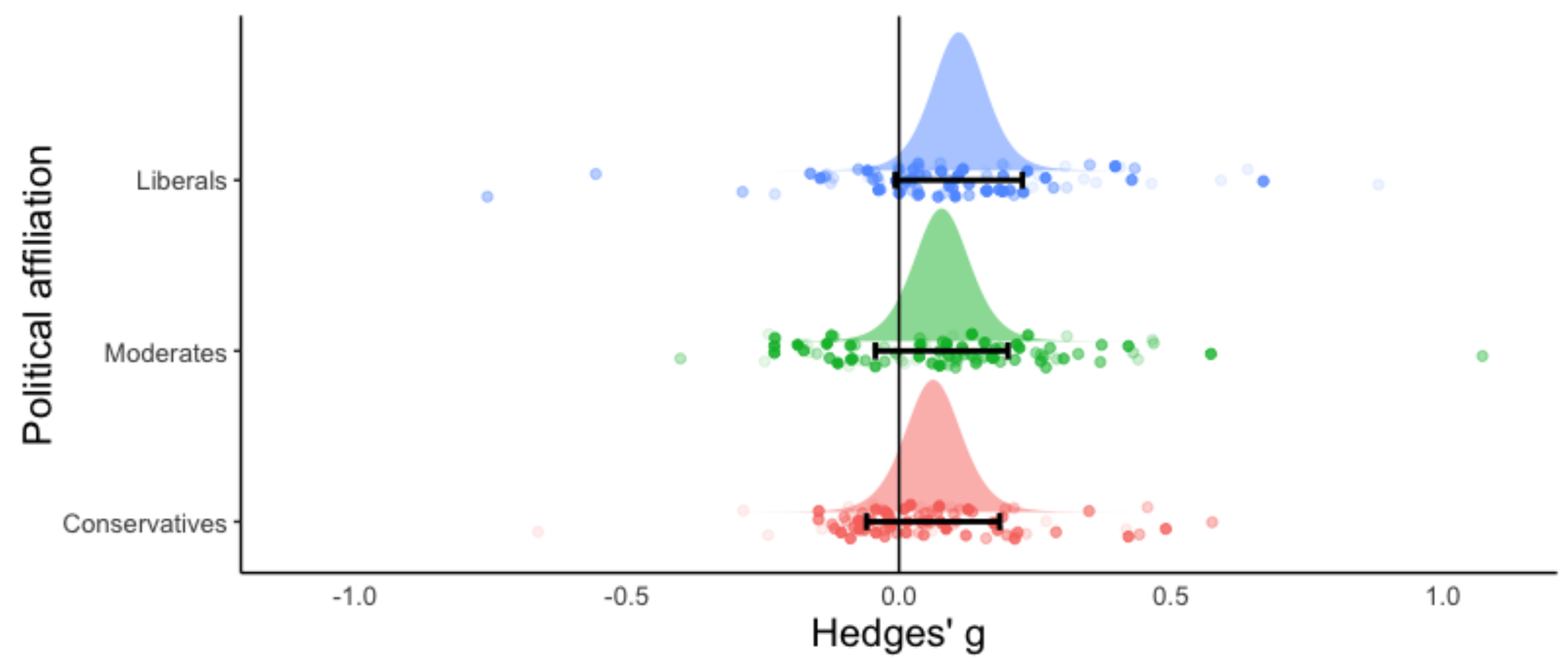

Note. The transparency of effect sizes reflects their weight (divided into ten weight classes). Error bars are $95 \%$ prediction intervals around the estimated effect size for each political group based on the meta-regression model. The $t$ distributions have a mean equal to the estimated effect size for each group and standard deviation equal to $\sqrt{S E^{2}+T^{2}}$. 
Table 1

Estimated Effect Sizes Split by Political Affiliation and Intervention Type

\begin{tabular}{|c|c|c|c|c|c|c|c|}
\hline Intervention type & $g$ & $d f$ & $95 \% \mathrm{CI}$ & $95 \% \mathrm{PI}$ & $p$ & $k$ & $n$ \\
\hline \multicolumn{8}{|l|}{ Consensus } \\
\hline Conservatives & 0.06 & 7.65 & {$[-0.003,0.13]$} & {$[-0.06,0.19]$} & .06 & 93 & 20 \\
\hline Moderates & 0.08 & 7.65 & {$[0.01,0.14]$} & {$[-0.04,0.20]$} & .02 & 93 & 20 \\
\hline Liberals & 0.11 & 7.65 & {$[0.06,0.16]$} & {$[-0.01,0.23]$} & .002 & 93 & 20 \\
\hline \multicolumn{8}{|l|}{ All other interventions } \\
\hline Conservatives & 0.08 & 26.68 & {$[0.02,0.13]$} & {$[-0.07,0.22]$} & .01 & 217 & 53 \\
\hline Moderates & 0.09 & 25.34 & {$[0.06,0.13]$} & {$[-0.04,0.23]$} & $<.001$ & 200 & 51 \\
\hline Liberals & 0.08 & 25.71 & {$[0.04,0.12]$} & {$[-0.06,0.22]$} & $<.001$ & 209 & 52 \\
\hline $\begin{array}{l}\text { Note. } g=\text { estimated eff } \\
\text { of independent sample } \\
(2009) \cdot p \text {-values indica } \\
\text { zero. Expanding to thr } \\
\text { effect sizes for non-cor }\end{array}$ & $\begin{array}{l}\text { size; } a \\
I=\text { pre } \\
f \text { the e }\end{array}$ & imatec & $\begin{array}{l}\text { es of freedom; } k \\
\text { terval, calculate } \\
\text { ffect size is sta }\end{array}$ & $\begin{array}{l}=\text { number of e } \\
\text { d using the for } \\
\text { istically signif }\end{array}$ & $\begin{array}{l}\text { ect size } \\
\text { aula in } \mathrm{E} \\
\text { antly di }\end{array}$ & $\begin{array}{l}n=n \\
\text { renst } \\
\text { erent }\end{array}$ & $\begin{array}{l}\text { aber } \\
\text { et al } \\
\text { m tive }\end{array}$ \\
\hline
\end{tabular}

\section{Discussion}

Given that recent studies suggest conservatives feel psychological reactance in response to consensus messages, we took advantage of meta-analytic data to evaluate the implications of this reactance on climate change attitudes. Though effect sizes were slightly larger for liberals than for conservatives, the difference was not statistically significant. Furthermore, the pattern of political differences was not statistically significantly different between consensus messages and other interventions, suggesting that consensus messages are not uniquely ineffective among conservatives.

How much of a role does psychological reactance play in consensus messaging studies? On one hand, it seems that consensus messages are not very effective for conservatives. This could be explained by the psychological reactance caused by these messages. Yet consensus messages are not very effective for liberals either, with no significant difference between the two 
groups. Either liberals are also feeling reactance to the messages, or consensus messages are limited in their overall effectiveness for people across the political spectrum. Importantly, consensus messages are not unique in their limited effectiveness, with effect sizes very similar to other interventions (and similar to the expected effect of consensus messages on downstream attitudes like policy support; van der Linden, 2021). ${ }^{3}$

We found no meta-analytic evidence that consensus messages backfire or negatively impact climate change attitudes for conservatives. This finding is in line with other research on political issues that finds counterattitudinal messages do not influence attitudes in the opposite direction of the message (Guess \& Coppock, 2020). Although research in motivated reasoning finds that being skeptical of counterattitudinal information is common among both liberals and conservatives (Ditto et al., 2019), there is a distinction between backfire-where counterattitudinal information actually strengthens pre-existing attitudes_-and resistance to counterattitudinal information. The current meta-analytic data seem to be more in line with resistance, where psychological reactance among conservatives is reflective of a typical response to counterattitudinal information, rather than the root of backfire.

The meta-analytic evidence should be interpreted in light of some limitations (see the original paper, for a longer discussion). First, the meta-analysis was not conducted for consensus messaging specifically, but for interventions more broadly. Therefore, we did not collect information specific to consensus messaging (e.g., perceived consensus). Second, the data are all from 2019 or earlier, leaving questions about the effectiveness of newer consensus messaging studies. Third, with only 20 independent samples, the current analysis had low statistical power to detect differences between groups. A potentially meaningful difference between conservative and liberal effect sizes could be statistically significant in an analysis with more statistical power, 
though the magnitude of difference across these 20 samples indicates that power alone is likely not driving the nonsignificant finding. Moreover, even a significant difference between liberals and conservatives would not necessarily imply backfire unless the estimated effect size was negative for conservatives. Fourth, as is the case with all meta-analyses, these aggregate data are unable to pinpoint specific combinations of factors potentially impacting effect sizes, potentially concealing situations where reactance has a larger or smaller effect on climate change attitudes.

Based on meta-analytic evidence from 20 studies, communication practitioners can be more confident that consensus messages about climate change likely will not backfire among conservatives. Since consensus messages can be shared in various ways and forums (e.g., videos, news articles; Goldberg et al., 2019; Rode, Iqbal, et al., 2021), it may be beneficial to make them more widespread. Yet consensus messages — similar to other persuasive messages — are unlikely to cause widespread changes in perspectives about divisive political topics (for a longer discussion, see Rode, Dent, et al., 2021; also see Coppock et al., 2020). Stronger effects may require messages to be particularly tailored to an audience or region (e.g., Goldberg et al., 2021). Alternatively, changing social norms may be a more powerful motivator than attempting to alter public opinion via persuasion (e.g., Sparkman et al., 2021).

In their recent study finding psychological reactance among conservatives, Chinn and Hart (2021) stated that, “...these findings suggest that well intentioned actors spreading consensus messages may inadvertently calcify positions against climate action" (p. 8). Through meta-analysis, we sought to provide context for this debate over consensus messaging by integrating previous research to inform a broader discussion about the existence and implications of reactance. Though the overall effectiveness of consensus messages can be debated-and it seems likely that such messages do arose feelings of reactance in some individuals - there is 
little evidence that consensus messages negatively impact conservatives' climate change attitudes. 


\section{Notes}

1. In the original paper, there were some overlaps between types of intervention (e.g., consensus and emotion) which required grouping some consensus interventions as other types (e.g., emotion). In the current analysis, since the focus is on consensus interventions, we included all interventions that included information about the scientific consensus.

2. In the original paper, we estimated the effect size for consensus messages alone and tested if political affiliation was a significant moderator of it. All other analyses in this paper are new (e.g., Figure 1, Table 1, estimating $g$ for each political group).

3. For a longer discussion about the importance of an effect size of this magnitude, see the original paper. 


\section{References}

Bayes, R., Bolsen, T., \& Druckman, J. N. (2020). A research agenda for climate change communication and public opinion: The role of scientific consensus messaging and beyond. Environmental Communication, 1-19. https://doi.org/10.1080/17524032.2020.1805343

Borenstein, M., Hedges, L. V., Higgins, J. P. T., \& Rothstein, H. R. (2009). Introduction to metaanalysis. Wiley.

Chinn, S., \& Hart, P. S. (2021). Climate change consensus messages cause reactance. Environmental Communication, 1-9. https://doi.org/10.1080/17524032.2021.1910530

Cook, J., Nuccitelli, D., Green, S. A., Richardson, M., Winkler, B., Painting, R., Way, R., Jacobs, P., \& Skuce, A. (2013). Quantifying the consensus on anthropogenic global warming in the scientific literature. Environmental Research Letters, 8(2), 024024. https://doi.org/10.1088/1748-9326/8/2/024024

Coppock, A., Hill, S. J., \& Vavreck, L. (2020). The small effects of political advertising are small regardless of context, message, sender, or receiver: Evidence from 59 real-time randomized experiments. Science Advances, 6(36), eabc4046. https://doi.org/10.1126/sciadv.abc4046

Ditto, P. H., Liu, B. S., Clark, C. J., Wojcik, S. P., Chen, E. E., Grady, R. H., Celniker, J. B., \& Zinger, J. F. (2019). At least bias is bipartisan: A meta-analytic comparison of partisan bias in liberals and conservatives. Perspectives on Psychological Science, 14(2), 273-291. https://doi.org/10.1177/1745691617746796

Dixon, G., Hmielowski, J., \& Ma, Y. (2019). More evidence of psychological reactance to consensus messaging: A response to van der Linden, Maibach, and Leiserowitz (2019). Environmental Communication, 1-7. https://doi.org/10.1080/17524032.2019.1671472 
Fisher, Z., \& Tipton, E. (2015). robumeta: An R-package for robust variance estimation in metaanalysis. http://arxiv.org/abs/1503.02220

Goldberg, M. H., Gustafson, A., Rosenthal, S. A., \& Leiserowitz, A. (2021). Shifting Republican views on climate change through targeted advertising. Nature Climate Change, 11, 573577. https://doi.org/10.1038/s41558-021-01070-1

Goldberg, M. H., van der Linden, S., Ballew, M. T., Rosenthal, S. A., Gustafson, A., \& Leiserowitz, A. (2019). The experience of consensus: Video as an effective medium to communicate scientific agreement on climate change. Science Communication, 41(5), 659673. https://doi.org/10.1177/1075547019874361

Guess, A., \& Coppock, A. (2020). Does counter-attitudinal Information cause backlash? Results from three large survey experiments. British Journal of Political Science, 50(4), 14971515. https://doi.org/10.1017/S0007123418000327

Hedges, L. V., Tipton, E., \& Johnson, M. C. (2010). Robust variance estimation in metaregression with dependent effect size estimates. Research Synthesis Methods, 1(1), 39-65. https://doi.org/10.1002/jrsm.5

Lakens, D. (2013). Calculating and reporting effect sizes to facilitate cumulative science: A practical primer for t-tests and ANOVAs. Frontiers in Psychology, 4. https://doi.org/10.3389/fpsyg.2013.00863

Landrum, A. R., \& Slater, M. H. (2020). Open questions in scientific consensus messaging research. Environmental Communication, 14(8), 1033-1046. https://doi.org/10.1080/17524032.2020.1776746

Leiserowitz, A., Maibach, E., Rosenthal, S., Kotcher, J., Carman, J., Wang, X., Goldberg, M., Lacroix, K., \& Marlon, J. (2020). Climate change in the American mind: December, 2020. 
New Haven, CT: Yale Project on Climate Change Communication.

https://climatecommunication.yale.edu/publications/climate-change-in-the-american-minddecember-2020/

Ma, Y., Dixon, G., \& Hmielowski, J. D. (2019). Psychological reactance from reading basic facts on climate change: The role of prior views and political identification. Environmental Communication, 13(1), 71-86. https://doi.org/10.1080/17524032.2018.1548369

Rains, S. A. (2013). The nature of psychological reactance revisited: A meta-analytic review. Human Communication Research, 39(1), 47-73. https://doi.org/10.1111/j.14682958.2012.01443.x

Rode, J. B., Dent, A. L., Benedict, C. N., Brosnahan, D. B., Martinez, R. L., \& Ditto, P. H. (2021). Influencing climate change attitudes in the United States: A systematic review and meta-analysis. Journal of Environmental Psychology, 76, 101623. https://doi.org/10.1016/j.jenvp.2021.101623

Rode, J. B., Iqbal, S., Butler, B. J., \& Ditto, P. H. (2021). Using a news article to convey climate science consensus information. Science Communication, 43(5), 651-673. https://doi.org/10.1177/10755470211027235

Sparkman, G., Howe, L., \& Walton, G. (2021). How social norms are often a barrier to addressing climate change but can be part of the solution. Behavioural Public Policy, 5(4), 528-555. https://doi.org/10.1017/bpp.2020.42

Tipton, E., \& Pustejovsky, J. E. (2015). Small-sample adjustments for tests of moderators and model fit using robust variance estimation in meta-regression. Journal of Educational and Behavioral Statistics, 40(6), 604-634. https://doi.org/10.3102/1076998615606099

van der Linden, S. (2021). The Gateway Belief Model (GBM): A review and research agenda for 
communicating the scientific consensus on climate change. Current Opinion in Psychology, 42, 7-12. https://doi.org/10.1016/j.copsyc.2021.01.005

van der Linden, S., Cook, J., Maertens, R., Goldberg, M., Kerr, J., Lewandowsky, S., Maibach, E., \& Leiserowitz, A. (2021). Do climate change consensus messages cause reactance? A comment on Chinn and Hart (2021). PsyArXiv. https://psyarxiv.com/kqsfz/ van der Linden, S., Maibach, E., \& Leiserowitz, A. (2019). Exposure to scientific consensus does not cause psychological reactance. Environmental Communication, 1-8. https://doi.org/10.1080/17524032.2019.1617763 